\title{
Racial Disparities in Colorectal Cancer Mortality: The Role of Endoscopy Wait-Time and Stage at Diagnosis
}

\author{
Rachel B. Issaka, MD, MAS ${ }^{1,2,3}$, Li Li, MA, MPA ${ }^{2}$, Catherine Fedorenko, MMSc ${ }^{2}$, Cynthia W. \\ Ko, MD, MS ${ }^{3}$, John M. Inadomi, MD $^{3}$, Scott D. Ramsey, MD, PhD $^{2}$ \\ ${ }^{1-}$ Clinical Research Division, Fred Hutchinson Cancer Research Center. Seattle, WA. USA. \\ ${ }^{2-}$ Hutchinson Institute for Cancer Outcomes Research, Fred Hutchinson Cancer Research \\ Center. Seattle, WA. USA. \\ ${ }^{3-}$ Division of Gastroenterology, University of Washington School of Medicine. Seattle, WA. USA.
}

\section{Abstract}

\begin{abstract}
Introduction-In Western Washington (WA), colorectal cancer (CRC) mortality between 20122016, was highest in American Indian/Alaska Natives (AI/AN) and African-Americans (AA) at 20.7 and 18.7 respectively compared to non-Hispanic Whites at 14.1/100,000 people. We hypothesized that time from billed encounters for CRC-associated symptoms to endoscopy completion or CRC stage at diagnosis contributed to observed differences.

Methods-Using administrative insurance claims linked to WA cancer registry data, we performed a retrospective cohort study of patients diagnosed with CRC between 2011-2017, with continuous insurance for 15-months prior to diagnosis and a billed encounter for CRC-associated symptoms. We determined the wait-time (days) and stage at diagnosis and conducted logistic regression analysis to identify the factors associated with endoscopy completion.
\end{abstract}

Results-Of the 3,461 CRC patients identified, 57\% had stage 2 or 3 disease with no differences in stage by race and $84 \%$ completed an endoscopy after a billed encounter for CRC-associated symptoms. The median wait-time to endoscopy was 52 days (IQR 14-218) without differences by

Terms of use and reuse: academic research for non-commercial purposes, see here for full terms. http://www.springer.com/gb/openaccess/authors-rights/aam-terms-v1

Corresponding Author: Rachel Issaka, MD, MAS, 1100 Fairview Ave. N., M/S: M3-B232, Seattle, WA 98109, rissaka@fredhutch.org, Office: (206) 667-1447, Fax: (206) 667-5977.

Author Contributions: Study concept and design: Issaka. Acquisition of data: Issaka, Li, Fedorenko. Statistical analysis: Issaka, Li. Drafting of the manuscript: Issaka. Critical revision of the manuscript for important intellectual content: All authors. Approval of the final manuscript All authors.

Conflict of Interest: No conflicts of interest were reported by the authors of this paper.

Guarantor of the article: Rachel Issaka, MD, MAS

Financial Disclosures: No financial disclosures were reported by the authors of this paper

Writing Assistance: None

Ethical approval: All procedures performed in studies involving human participants were in accordance with the ethical standards of the institutional research committee (Fred Hutchinson Institutional Review Board, IR File \#8694) and with the 1964 Helsinki declaration and its later amendments or comparable ethical standards.

Publisher's Disclaimer: This Author Accepted Manuscript is a PDF file of a an unedited peer-reviewed manuscript that has been accepted for publication but has not been copyedited or corrected. The official version of record that is published in the journal is kept up to date and so may therefore differ from this version. 
race. Compared to patients diagnosed with stage $1 \mathrm{CRC}$, patients with stage $4 \mathrm{CRC}$ were more likely to complete an endoscopy within the first quartile of time ( $22.2 \%$ vs. $17.4 \%, \mathrm{p}<0.01)$. Living arrangement, insurance type and comorbidity, but not race, were significant factors associated with endoscopy completion.

Conclusions-We found no statistically significant differences in time from billed CRCassociated symptoms to endoscopy completion or in CRC stage among AA and AI/AN compared to Whites. This suggests other factors are more likely to contribute to observed mortality disparities.

\section{Keywords}

Colorectal Cancer; Disparities; Administrative Claims; Cancer Registry

\section{Introduction}

Colorectal cancer (CRC) is the $3^{\text {rd }}$ most common cancer and second-leading cause of cancer death in Washington (WA) state.[1] In Western WA, between 2012 and 2016, CRC-mortality was highest in American Indian/Alaska Natives (AI/AN) and African-Americans (AA) at 20.7 and 18.7 respectively compared to non-Hispanic Whites at 14.1 per 100,000 people. Among racial and ethnic minorities, the impact of lack of access to cancer care is well documented[2] and the contributing factors are multifactorial.[3, 4] While the impact of later stage disease at diagnosis is improving for $\mathrm{AI} / \mathrm{AN}[5]$ and $\mathrm{AA},[6]$ nationally, lack of screening remains a frequently cited contributor to racial and ethnic CRC-mortality disparities. Per the recent Behavioral Risk Factor Surveillance System - a national healthrelated telephone survey $-70 \%$ of all eligible adults in the United States reported being upto-date with CRC screening. These values were modestly higher in WA state where $72 \%$ of all eligible adults and $68 \%$ and $66 \%$ of $\mathrm{AA}$ and $\mathrm{AI} / \mathrm{AN}$, respectively, reported being up-todate with CRC screening.[7] While over-reporting of screening is more common in surveys compared to review of electronic health records, [8] these data suggest other factors beyond screening participation may contribute to CRC-mortality disparities in WA state.

In addition to lack of screening, diagnostic delays, including lack of utilization of health care services and inadequate follow-up after symptom onset are potential contributors to national CRC-mortality disparities across race and ethnicity.[9] However, the impact of delayed symptom follow-up on CRC-mortality disparities in the state of WA is unknown. Therefore, the aim of this study was to explore if lack of diagnostic endoscopy among patients with symptoms suggestive of CRC contributed to racial CRC-mortality disparities within WA state. Using administrative claims linked to WA cancer registry data, we sought to determine if differences in time from billed CRC-associated symptoms to endoscopy completion (hereon referred to as wait-time) between AA and AI/AN compared to Whites may contribute to mortality disparities. Secondarily, we also aimed to determine if CRC stage at time of diagnosis by race contributed to these observed differences. Finally, we sought to identify factors associated with endoscopy completion among patients with billed encounters for CRC-associated symptoms. 


\section{Methods}

\section{Study Design and Population}

We conducted a retrospective cohort study of CRC cases diagnosed from January 1, 2011 to December 31, 2017 that represented an individual's first malignant disease in the Western Washington Cancer Surveillance System (CSS) or the Washington State Cancer Registry (WSCR). As the present analysis sought to determine the impact of diagnostic delays, adults 20 years and older whose first malignant disease was CRC were included. Patients with symptoms suggestive of CRC for 15-months or less prior to diagnosis and continuous insurance coverage for the same time period were also included. The 15-month time period was selected due to recent evidence that the Charlson Score may continue to predict mortality within this time period fairly well[10] and lack of substantive differences in our 12-month and 15-month study populations. Patients with documented American Joint Committee on Cancer (AJCC) stage 0 or unstaged disease, CRC symptoms present for more than 15-months prior to diagnosis, or whose symptom date occurred after initial endoscopy were excluded (Figure 1).

\section{Cohort Identification}

We used the International Classification of Diseases, Ninth and Tenth Revisions (ICD-9/10), Current Procedural Terminology (CPT), and Healthcare Common Procedure Coding System (HCPCS) codes (Appendix Table 1) to identify the occurrence and dates of CRC-associated symptoms in relation to colonoscopy and flexible sigmoidoscopy (hereon referred to as endoscopy) completion. CRC-associated symptoms included were abdominal pain, abdominal mass, abnormal feces/change in bowel habits, anemia (iron-deficiency and nototherwise-specified), constipation, diarrhea, gastrointestinal bleeding and weight loss (Appendix Table 1). We included all CRC-symptoms submitted for administrative claims prior to diagnosis, thus allowing for multiple symptoms per patient in some instances. Endoscopies spanning an 18-month period (15-months prior to CRC diagnosis to account for symptom triggered endoscopic evaluation and 3-months after CRC diagnosis to account for delays in claims submission), were included.

\section{Data Sources}

Data from Hutchinson Institute for Cancer Outcomes Research (HICOR) was used to identify the patient population. The HICOR data repository consists of health plan enrollment and claims files linked to cancer registry records from CSS and WSCR. Health plan participants in the enrollment and claims files include two large commercial insurers (Premera Blue Cross and Regence Blue Shield), Washington State Public Employees Benefits Board's Uniform Medical Plan (WA Medicaid), and Medicare. Enrollment and claims files were available for all insurers through December 31, 2018. This HICOR collective data asset represents approximately 70 percent of cancer patients who received cancer care in Washington State during the study time period.[11] CSS collects comprehensive information on staging, treatment, and survival for those diagnosed with malignancies in 13 Western Washington counties, excluding non-melanoma skin cancer. The CSS is part of the National Cancer Institute Surveillance, Epidemiology, and End Results 
(SEER) program and also provides data to WSCR. WSCR monitors cancer incidence and mortality in the entire state of Washington.

\section{Ascertainment of Covariates}

Our dependent variables were endoscopy completion, median wait-time to endoscopy completion and stage at diagnosis. Our independent variables included demographics (age, sex, race, ethnicity, insurance type, living arrangement), socioeconomic status measured by median income according to zip code of residence using 2015 Census American Community Survey Data, rural/urban residence based on 2010 Rural-Urban Commuting Area Codes Maps (RUCA), AJCC stage, and an adaptation of the Charlson Comorbidity Index developed for use with SEER-Medicare data.[12] Insurance type was classified as Commercial, Medicare, Medicaid or Multiple if individuals had two of the three insurance types. Finally, living arrangement was classified as living with a partner, living without a partner or unknown.

\section{Statistical Analysis}

Patient demographic information was described as proportions or medians and interquartile ranges (IQR). Days from billed CRC-associated symptom to endoscopy completion (i.e. wait-time) was described using medians and IQR. Differences between groups were assessed using $\chi^{2}$, student's t-test, and nonparametric median two-sample test as appropriate. For our primary outcome, we compared median wait-times between Whites and AA, AI/AN and Asians/PI respectively using the Wilcoxon rank sum test. Secondarily, we examined stage at diagnosis by race and performed logistic regression analysis to determine the factors associated with receipt of endoscopy after a billed CRC-associated symptom. We also tested the outcome of late endoscopy completion ( $\geq 180 \mathrm{~d}$ ) compared to early endoscopy completion in a logistic regression model as literature suggests a significantly higher risk for CRC when screening tests follow-up occur after 6-months.[13] Accompanying odds ratios (OR), 95\% confidence interval (CI), and $\mathrm{P}$ values were reported in all instances and $\mathrm{P}$ values $<0.05$ were considered statistically significant. We used Stata/SE (version 15.0; StataCorp LP, College Station, TX, USA) statistical software for all analyses.

\section{Results}

Based on inclusion and exclusion criteria, 3,461 patients were eligible for the study (Figure 1). The median age was 72 years, $52 \%(\mathrm{n}=1,792)$ were female, $90 \%$ White, $6 \%$ Asian/PI, $2 \% \mathrm{AA}$ and $1 \% \mathrm{AI} / \mathrm{AN} ; 3 \%$ were Hispanic, $74 \%(\mathrm{n}=2,548)$ had colon cancer with the remaining diagnosed with rectal cancer, and $54 \%(\mathrm{n}=1,862)$ had a Charlson Comorbidity Index (CCI) score of 0 . Of the patients that met inclusion criteria, $84 \%(n=2,909)$ completed an endoscopy (colonoscopy $n=2,704$; flexible sigmoidoscopy $n=205$ ) after a billed encounter for a CRC-associated symptom (Table 1 ). The majority $(57 \%, \mathrm{n}=1,982)$ were diagnosed with AJCC stage 2 or 3 CRC.

We found no differences in CRC stage at diagnosis among AA and AN/AI compared to Whites (Table 2). We also found no differences in early-stage CRC (stage I/II) or late-stage CRC (stage III/IV) by race (Appendix Table 2). The median wait-time between any CRC- 
associated symptom and endoscopy for all patients was 52 days (IQR 14-218). Compared to Whites, there was no statistically significant difference in median wait-times among AA (60d vs. 51d; p=0.96), AI/AN (65d vs. 51d; $\mathrm{p}=0.59$ ) and Asian/PI (63d vs. 51d; $\mathrm{p}=0.24$ ) respectively. In a logistic regression model testing the outcome of late endoscopy completion ( $\geq 180 \mathrm{~d}$ ) compared to early endoscopy completion $(<180)$, women were more likely to complete a late endoscopy compared to men $(32 \%$ vs $26 \%$, OR 1.35 , CI $1.14-1.60$, p $<0.01)$ as were patients with a CCI score of $2+$ compared to patients with CCI score of 0 ( $44 \%$ vs. $21 \%$, OR 2.72, CI 2.22-3.33, p<0.01) (data not shown). There was no difference by race among patients who completed an early or late endoscopy for CRC-associated symptoms.

In our analysis, the most frequently billed symptoms were anemia $(n=1060)$, gastrointestinal bleeding ( $\mathrm{n}=995)$ and abdominal pain $(\mathrm{n}=691)$. Overall, abdominal mass was associated with the shortest median wait-time (9.5d) while diarrhea and anemia led to the longest median wait-time (67d and 74.5d) (Table 3). Compared to CCI scores of 0 , patients with CCI scores of 1 and $2+$ had longer median wait-times, $39 \mathrm{~d}$ vs. $62 \mathrm{~d}(<0.01)$ and $39 \mathrm{~d}$ vs. $128 \mathrm{~d}(\mathrm{p}<0.01)$. Additionally, compared to patients diagnosed with stage 1 CRC, patients with stage 4 CRC were more likely to complete an endoscopy within the first quartile of time (22.2 vs. $17.4 \%$, $\mathrm{p}<0.01)$ and less likely to complete an endoscopy in the fourth quartile of time $(13.4 \%$ vs. $24.4 \%, \mathrm{p}<0.01)$.

Univariate logistic regression revealed that patients 65 years and older were less likely to complete an endoscopy than patients under 65 years (83\% vs. 88\%, OR 0.65 , CI $0.53-0.81$, $\mathrm{p}<0.01)$ as were patients with Medicare insurance compared to commercially insured patients ( $81 \%$ vs. $89 \%$, OR 0.53 , CI $0.42-0.68, \mathrm{p}<0.01)$. Also, patients with CCI score of $2+$ were less likely to complete an endoscopy compared to patients with CCI score of 0 (79\% vs. $86 \%$, OR 0.62 , CI $0.50-0.76, \mathrm{p}<0.01$ ) while those living with a partner were more likely to complete an endoscopy compared to those living without a partner ( $87 \%$ vs. $80 \%$, OR 1.63 , CI 1.34-1.98, $\mathrm{p}<0.01$ ). There were no statistical differences in endoscopy completion by gender, race, ethnicity, RUCA score, or socioeconomic status.

Our final multivariate logistic model adjusted for gender, race, ethnicity, insurance type, living arrangement, Charlson Comorbidity Index score ( 0,1 , or $2+)$, RUCA, and socioeconomic status.

Age was not included as we found a strong correlation between age and insurance type $(\mathrm{R}=0.7)$. In this model, living with a partner (OR 1.52, CI 1.23-1.86, $\mathrm{p}<0.01)$, Medicare insurance (OR 0.63, CI 0.48-0.82, p=0.01), and comorbidity score of 2+ (OR 0.71, CI 0.57$0.88, \mathrm{p}<0.01)$ remained strongly associated with endoscopy completion after a billed encounter for CRC-associated symptoms (Table 4).

\section{Discussion}

Historically, racial and ethnic minority groups including AA and AI/AN have had less access to care which contributes to higher disease CRC-mortality.[2] However, as CRC represents a continuum of care beginning with risk assessment until surveillance,[14] in this retrospective analysis of persons with insurance, we sought to examine the potential impact 
of diagnostic delays and CRC stage at diagnosis by race. Ultimately, we found the wait-time from billed CRC-associated symptoms to endoscopy completion was a median of 52 days and did not differ between minority racial groups. We also observed no significant differences in CRC stage distribution at diagnosis among AA and AI/AN compared to Whites in our cohort.

International data on the association of diagnostic delays and stage at diagnosis for patients with CRC are conflicting. For example, several Canadian studies have reported no association between wait-time and CRC-stage at diagnosis. However, these studies defined wait-times as the time from primary care provider referral to gastroenterology, first endoscopy or surgery.[15, 16] Other studies report longer wait-times associated with later stages of rectal cancer but earlier stages for colon cancer or a U-shaped relationship with higher mortality among patients with the shortest and longest delays.[17, 18] These findings are attributed to method limitations, including analyzing colon and rectal cancers together, differential definitions of wait-times, or failing to control for tumor grade which can confound results.[19] Despite these inconsistencies, it is possible that wait-time does not adversely affect patient outcomes due to the slow-growing nature of CRC.[20] We found no comparable studies that examined wait-times from billed encounters for CRC-associated symptoms to endoscopy completion, an outcome obtainable from administrative claims data. Additionally, we found no comparable studies that examined differences in these wait-times by race or ethnicity as a potential mechanism to explain CRC-mortality disparities.

Supporting our finding of no stage differences by race, May et al found that between 1975 and 2012, late-stage CRC declined more substantially among AA vs non-Hispanic Whites (by $0.45 \%$ and $0.27 \%$, respectively), despite persistently higher CRC-incidence among AA compared to Whites.[21] In their study, they postulate that the discordance in persistently higher CRC incidence among AA with yet declining stage-disparities may be attributed to increased utilization of CRC screening and inadequate modification of CRC risk-exposing factors (e.g. obesity). For AI/AN Medicaid enrollees, prior studies have demonstrated that race was not associated with later CRC-stage at diagnosis,[22] suggesting that CRC outcome differences in the broader population may be due to disparities in healthcare access.

Disparities in access to healthcare could lead to delays between symptom onset and clinician evaluation and more advanced disease within stages. Additionally, differential clinician recording and thus billing of CRC-symptoms by race could also lead to a lack of association between stage and race in our cohort. Neither of these potentially influential factors are captured by our study.

We found that older age, Medicare insurance, and higher CCI scores were inversely related to endoscopy completion after a billed encounter for CRC-associated symptoms. This likely reflects a growing body of literature suggesting that endoscopic evaluation among older adults with comorbidities, limited life expectancy and previous normal endoscopic evaluation may cause more harm than benefit.[23, 24] Additionally, decreased endoscopic evaluation in older patients may be due to Medicare cost-sharing requirements[25] and time needed to coordinate care given comorbidities. According to a report by the Kaiser Family Foundation, AA Medicare beneficiaries compared to White Medicare beneficiaries, were less likely to visit an office-based physician ( $71 \%$ vs. $80 \%)$ but more likely to have an 
inpatient stay (21\% vs. $19 \%)$ and visit the emergency room (37\% vs. $28 \%)$.[26] While similar data was not available for $\mathrm{AI} / \mathrm{AN}$, it is possible that cost-sharing requirements contribute to these findings and more adversely affects older AA and AI/AN. While our study did not find differences in endoscopy completion by race, the impact of Medicare costsharing should be explored in settings where these differences exist as unaffordable costs could lead to delayed symptom evaluation and later-stage CRC diagnosis. Similar to CRC screening literature, living with a partner was directly associated with endoscopy completion. Several reasons for this association noted in screening apply to this context as well, including the emotional support provided by a partner and reduced logistical burden of coordinating endoscopy scheduling and completion.[27]

There are several strengths to the present analysis. First, our study included a cohort of persons with insurance with comprehensive health plan enrollment and claims files linked to cancer registry records from Washington state. This data asset represents approximately $70 \%$ of cancer patients who received care in the state of Washington during the study time period. Second, our time period after billed CRC-symptoms (15-months prior to and 3-months after CRC diagnosis) enabled us to capture a maximal number of endoscopic procedures and accounted for delays in claims submission.

Several limitations are also worth noting. First, as this is a retrospective cohort study, the quality of the data is limited to quality of administrative claims reporting, particularly for symptoms, and we were unable to determine the impact of lack of insurance on our outcomes of interest. Second, there was limited representation of racial and ethnic minorities, specifically our populations of interest, AA and AI/AN, limiting statistical power to detect differences between racial groups. Despite this limitation, representation of racial and ethnic minorities in our cohort, mirrored those reported by the US Census Bureau for the state of Washington in 2018,[28] in which 79\% of the Washingtonians self-identified as White, $4.3 \%$ as AA, and $1.9 \%$ as AI/AN. Additionally, the observed differences in waittimes between racial groups were modest and unlikely to result in more advanced stage disease at diagnosis. Third, patients with supplemental insurance plans not through Premera Blue Cross, Regence Blue Shield, WA Medicaid or Medicare may not have been completely captured in our cohort.

In conclusion, our analyses revealed that among CRC patients in Western WA, there were no differences in wait-time and stage at diagnosis among AA and AI/AN when compared to Whites. This suggests that other factors including differential access to care, delays in symptom onset to medical evaluation, differences in treatment after diagnosis, and inadequate surveillance after treatment may potentially contribute to observed mortality disparities and are worth further exploration.

\section{Supplementary Material}

Refer to Web version on PubMed Central for supplementary material. 


\section{Acknowledgements}

Grant Support: This research was supported by the National Institutes of Health/National Cancer Institute Cancer Center Support Grant P30 CA015704. The funders had no role in the study design, data collection and analysis, decision to publish, or preparation of the manuscript.

\section{References}

1. Siegel RL, Miller KD, Jemal A. Cancer statistics, 2019. CA Cancer J Clin. 2019;69(1):7-34. doi:10.3322/caac.21551. [PubMed: 30620402]

2. Yabroff KR, Gansler T, Wender RC, Cullen KJ, Brawley OW. Minimizing the burden of cancer in the United States: Goals for a high-performing health care system. CA Cancer J Clin. 2019;69(3):166-83. doi:10.3322/caac.21556. [PubMed: 30786025]

3. Williams R, White P, Nieto J, Vieira D, Francois F, Hamilton F. Colorectal Cancer in African Americans: An Update. Clin Transl Gastroenterol. 2016;7(7):e185. doi:10.1038/ctg.2016.36. [PubMed: 27467183]

4. Guadagnolo BA, Petereit DG, Coleman CN. Cancer Care Access and Outcomes for American Indian Populations in the United States: Challenges and Models for Progress. Semin Radiat Oncol. 2017;27(2):143-9. doi:10.1016/j.semradonc.2016.11.006. [PubMed: 28325240]

5. Burnett-Hartman AN, Adams SV, Bansal A, McDougall JA, Cohen SA, Karnopp A et al. Indian Health Service Care System and Cancer Stage in American Indians and Alaska Natives. J Health Care Poor Underserved. 2018;29(1):245-52. doi:10.1353/hpu.2018.0017. [PubMed: 29503298]

6. White A, Joseph D, Rim SH, Johnson CJ, Coleman MP, Allemani C. Colon cancer survival in the United States by race and stage (2001-2009): Findings from the CONCORD-2 study. Cancer. 2017;123 Suppl 24:5014-36. doi:10.1002/cncr.31076. [PubMed: 29205304]

7. Centers for Disease Control and Prevention. National Center for Chronic Disease Prevention and Health Promotion, Division of Population Health. BRFSS Prevalence \& Trends Data 2015 https:// www.cdc.gov/brfss/brfssprevalence/. Accessed February 32020.

8. Rauscher GH, Johnson TP, Cho YI, Walk JA. Accuracy of self-reported cancer-screening histories: a meta-analysis. Cancer Epidemiol Biomarkers Prev. 2008;17(4):748-57. doi:10.1158/1055-9965.EPI-07-2629. [PubMed: 18381468]

9. Lee W, Nelson R, Mailey B, Duldulao MP, Garcia-Aguilar J, Kim J. Socioeconomic factors impact colon cancer outcomes in diverse patient populations. J Gastrointest Surg. 2012;16(4):692-704. doi:10.1007/s11605-011-1809-y. [PubMed: 22258868]

10. Berg GD, Gurley VF. Development and validation of 15 -month mortality prediction models: a retrospective observational comparison of machine-learning techniques in a national sample of Medicare recipients. BMJ Open. 2019;9(7):e022935. doi:10.1136/bmjopen-2018-022935.

11. HICOR. Community Cancer Care in Washington State Quality and Cost Report 2018. Fred Hutchinson Cancer Research Center, Seattle, WA 2018 https://www.fredhutch.org/content/dam/ public/labs-projects/hicor/CCCReport/HICOR\%20Community\%20Cancer\%20Care\%20Report \%20May\%202018.pdf. Accessed December 52018.

12. Healthcare Delivery Research Program NCI. National Cancer Institute comorbidity index overview. https://healthcaredelivery.cancer.gov/seermedicare/considerations/comorbidity.html. Accessed Feb 072019.

13. Corley DA, Jensen CD, Quinn VP, Doubeni CA, Zauber AG, Lee JK et al. Association Between Time to Colonoscopy After a Positive Fecal Test Result and Risk of Colorectal Cancer and Cancer Stage at Diagnosis. JAMA. 2017;317(16):1631-41. doi:10.1001/jama.2017.3634. [PubMed: 28444278]

14. Zapka JG, Taplin SH, Solberg LI, Manos MM. A framework for improving the quality of cancer care: the case of breast and cervical cancer screening. Cancer Epidemiol Biomarkers Prev. 2003;12(1):4-13. [PubMed: 12540497]

15. Janssen RM, Takach O, Nap-Hill E, Enns RA. Time to Endoscopy in Patients with Colorectal Cancer: Analysis of Wait-Times. Can J Gastroenterol Hepatol. 2016;2016:8714587. doi:10.1155/2016/8714587. [PubMed: 27446872] 
16. Singh H, Shu E, Demers A, Bernstein CN, Griffith J, Fradette K. Trends in time to diagnosis of colon cancer and impact on clinical outcomes. Can J Gastroenterol. 2012;26(12):877-80. [PubMed: 23248786]

17. Ramos M, Esteva M, Cabeza E, Llobera J, Ruiz A. Lack of association between diagnostic and therapeutic delay and stage of colorectal cancer. Eur J Cancer. 2008;44(4):510-21. doi:10.1016/ j.ejca.2008.01.011. [PubMed: 18272362]

18. Torring ML, Frydenberg M, Hamilton W, Hansen RP, Lautrup MD, Vedsted P. Diagnostic interval and mortality in colorectal cancer: U-shaped association demonstrated for three different datasets. J Clin Epidemiol. 2012;65(6):669-78. doi:10.1016/j.jclinepi.2011.12.006. [PubMed: 22459430]

19. Pruitt SL, Harzke AJ, Davidson NO, Schootman M. Do diagnostic and treatment delays for colorectal cancer increase risk of death? Cancer causes \& control : CCC. 2013;24(5):961-77. doi:10.1007/s10552-013-0172-6. [PubMed: 23446843]

20. Meester RG, Zauber AG, Doubeni CA, Jensen CD, Quinn VP, Helfand M et al. Consequences of Increasing Time to Colonoscopy Examination After Positive Result From Fecal Colorectal Cancer Screening Test. Clin Gastroenterol Hepatol. 2016;14(10):1445-51 e8. doi:10.1016/ j.cgh.2016.05.017. [PubMed: 27211498]

21. May FP, Glenn BA, Crespi CM, Ponce N, Spiegel BMR, Bastani R. Decreasing Black-White Disparities in Colorectal Cancer Incidence and Stage at Presentation in the United States. Cancer Epidemiol Biomarkers Prev. 2017;26(5):762-8. doi:10.1158/1055-9965.EPI-16-0834. [PubMed: 28035021]

22. Adams SV, Burnett-Hartman AN, Karnopp A, Bansal A, Cohen SA, Warren-Mears V et al. Cancer Stage in American Indians and Alaska Natives Enrolled in Medicaid. Am J Prev Med. 2016;51(3):368-72. doi:10.1016/j.amepre.2016.02.016. [PubMed: 27020318]

23. Day LW, Kwon A, Inadomi JM, Walter LC, Somsouk M. Adverse events in older patients undergoing colonoscopy: a systematic review and meta-analysis. Gastrointest Endosc. 2011;74(4):885-96. doi:10.1016/j.gie.2011.06.023. [PubMed: 21951478]

24. Issaka RB, Inadomi JM. Low-Value Colorectal Cancer Screening: Too Much of a Good Thing? JAMA Netw Open. 2018;1(8):e185445. doi:10.1001/jamanetworkopen.2018.5445. [PubMed: 30646272]

25. Osborn R, Moulds D, Squires D, Doty MM, Anderson C. International survey of older adults finds shortcomings in access, coordination, and patient-centered care. Health Aff (Millwood). 2014;33(12):2247-55. doi:10.1377/hlthaff.2014.0947. [PubMed: 25410260]

26. Kaiser Family Foundation. Profile of Medicare Beneficiaries by Race and Ethnicity: A Chartpack. 2016 https://www.kff.org/medicare/report/profile-of-medicare-beneficiaries-by-race-and-ethnicitya-chartpack/. Accessed February 32020.

27. El-Haddad B, Dong F, Kallail KJ, Hines RB, Ablah E. Association of marital status and colorectal cancer screening participation in the USA. Colorectal Dis. 2015;17(5):O108-14. doi:10.1111/ codi.12926. [PubMed: 25704636]

28. Bureau USC. Quick Facts. 2018 https://www.census.gov/quickfacts/map/IPE120213/53. Accessed November 12019. 


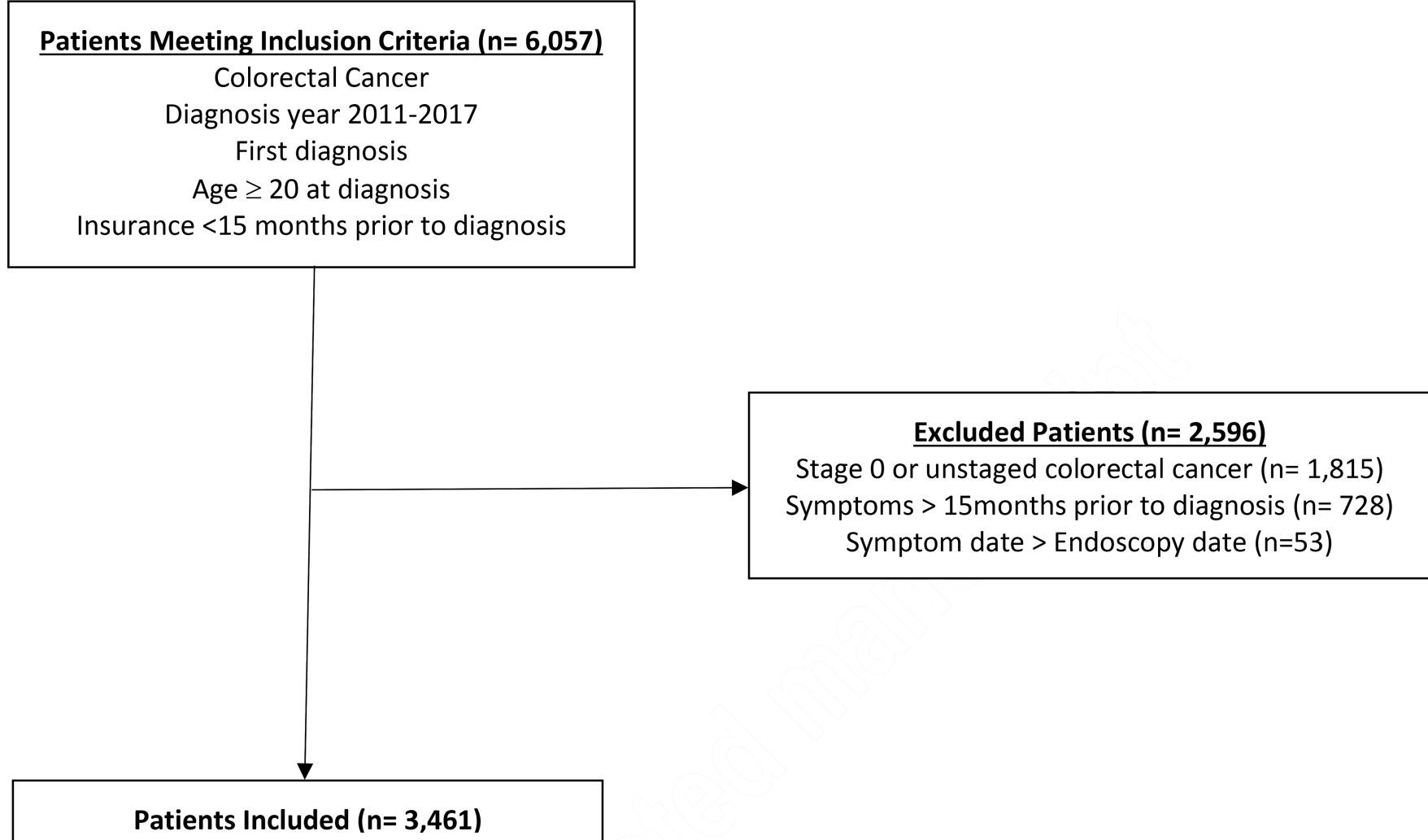

Figure 1:

Flow diagram of included and excluded patients 


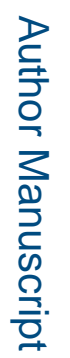

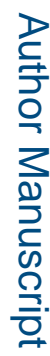

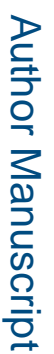

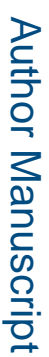

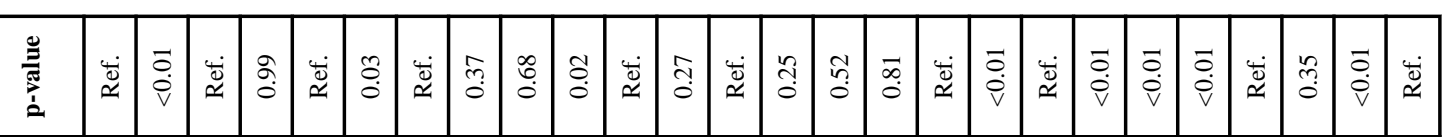

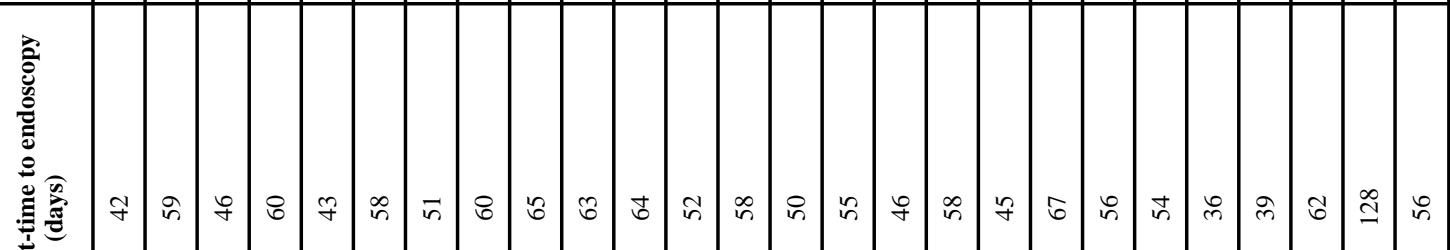

\section{赔}

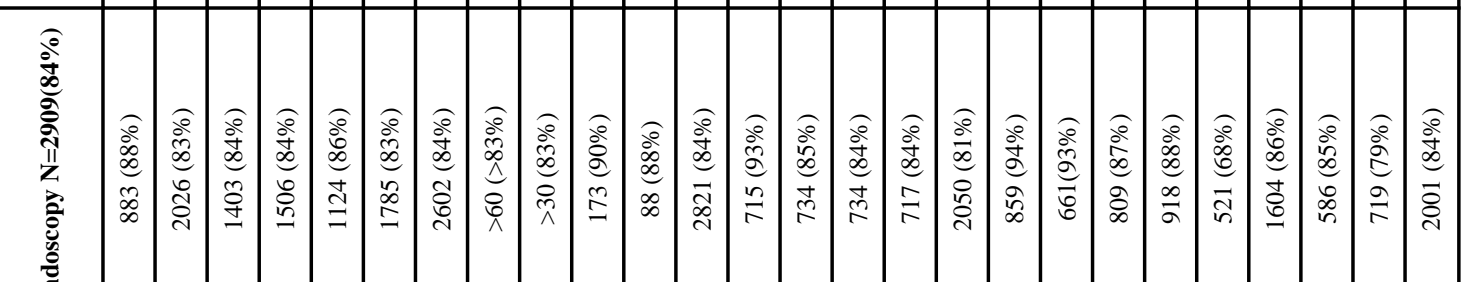

焉

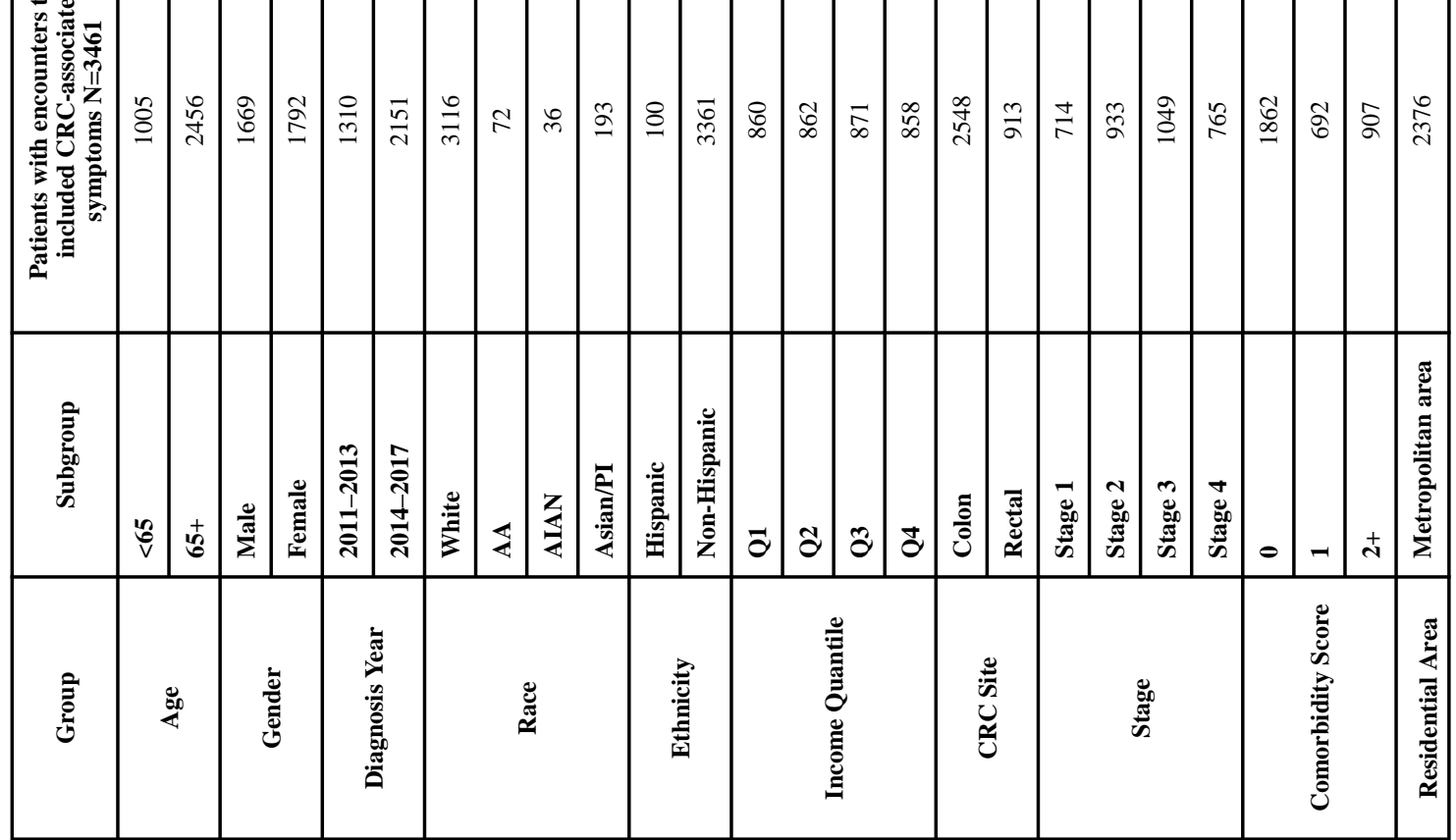

J Racial Ethn Health Disparities. Author manuscript; available in PMC 2021 October 01. 


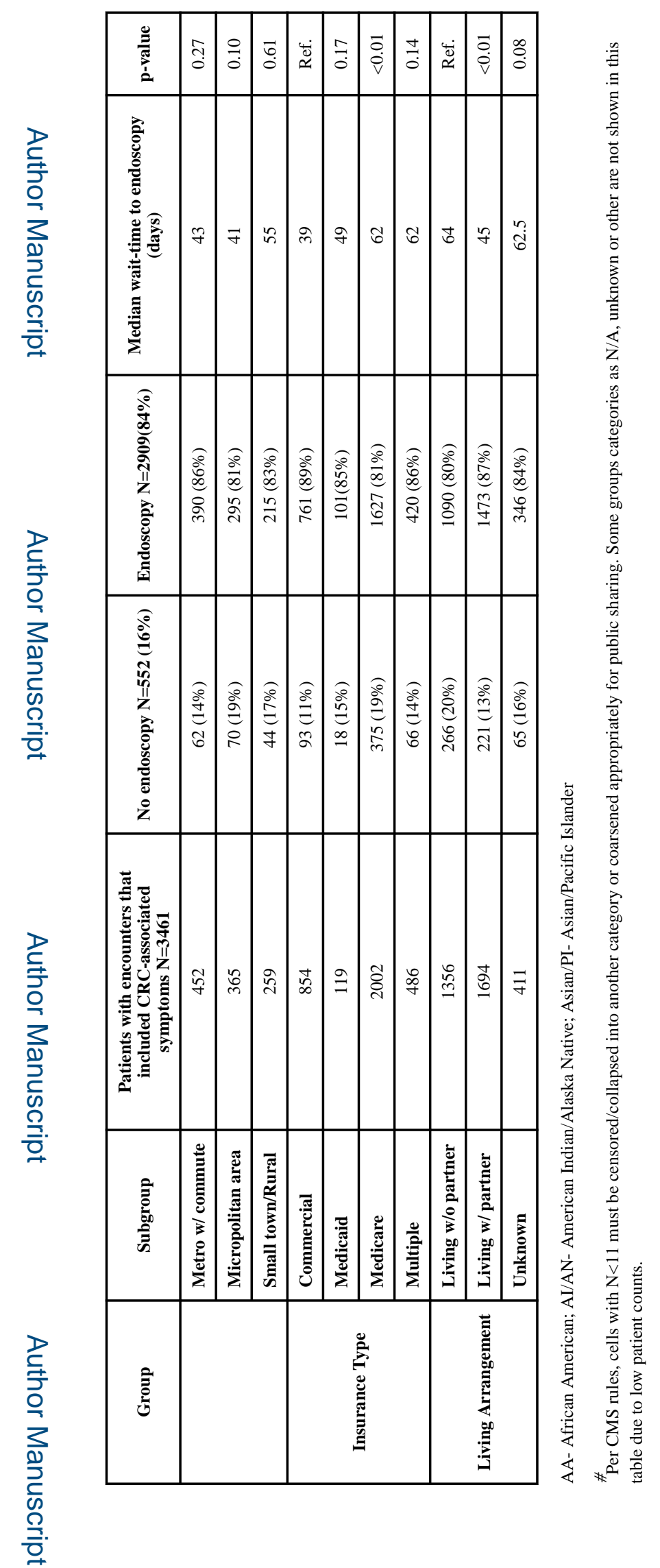

J Racial Ethn Health Disparities. Author manuscript; available in PMC 2021 October 01. 


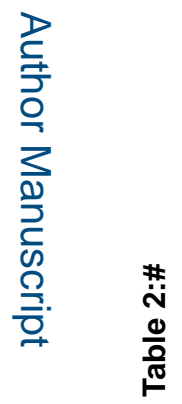

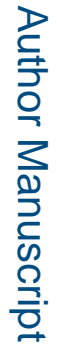

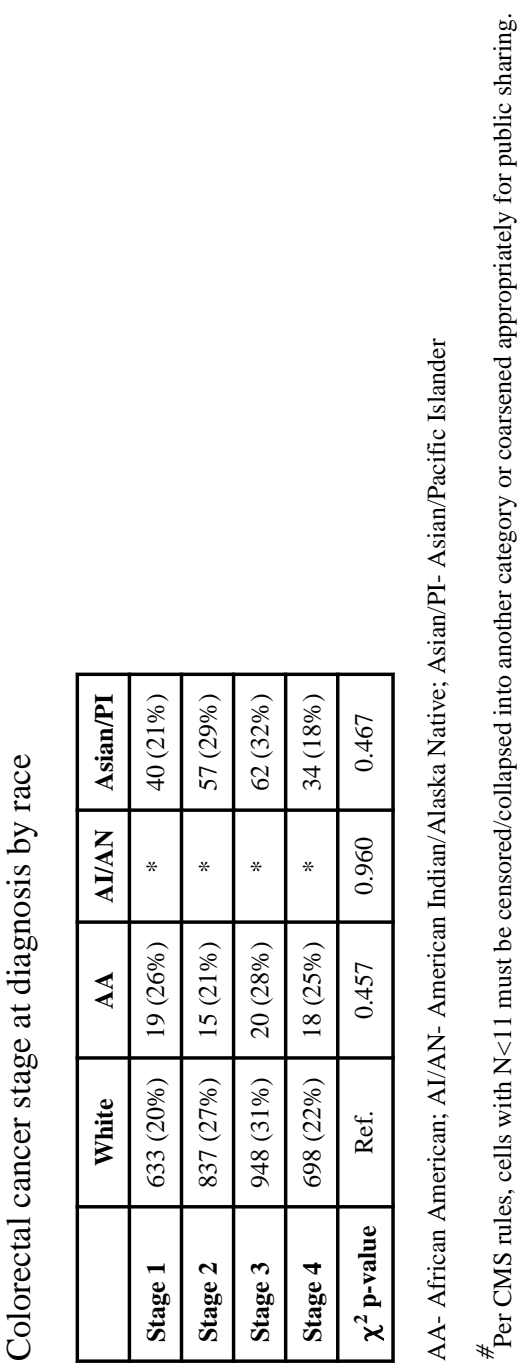

J Racial Ethn Health Disparities. Author manuscript; available in PMC 2021 October 01. 


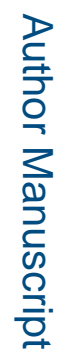

\begin{tabular}{|c|c|c|c|c|c|c|c|c|c|c|c|c|}
\hline \multirow{2}{*}{ 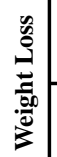 } & : & i & ल) & $\pi$ & 2 & $\hat{m}$ & $\stackrel{\sim}{\pi}$ & * & * & 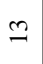 & * & $\grave{\lambda}$ \\
\hline & $z$ & $\Xi$ & $\stackrel{\infty}{+}$ & I & 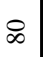 & \& & $\underline{n}$ & * & * & $=$ & * & 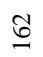 \\
\hline \multirow{2}{*}{ 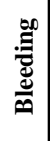 } & 量 & $\stackrel{L}{\pi}$ & $\bar{\lambda}$ & $\underset{\sim}{\Delta}$ & $\underset{\sim}{\infty}$ & i & $\stackrel{\sim}{\circ}$ & 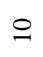 & $\bar{\lambda}$ & $\bar{m}$ & $\hat{m}$ & $\stackrel{i}{1}$ \\
\hline & $z$ & $\stackrel{\circ}{\circ}$ & ర్ల & రై & in & 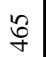 & D) & $=$ & $\cong$ & $\infty$ & le & ूू \\
\hline \multirow{2}{*}{ 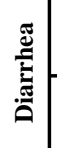 } & : & 5 & in & 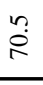 & 8 & 8 & 5 & $*$ & * & $\stackrel{\infty}{\sim}$ & * & $\delta$ \\
\hline & $z$ & $\stackrel{ }{m}$ & \& & $\stackrel{\circ}{N}$ & I & $\stackrel{\infty}{\circ}$ & ه् & * & * & $=$ & * & ర్ల \\
\hline
\end{tabular}

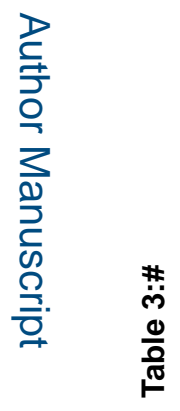

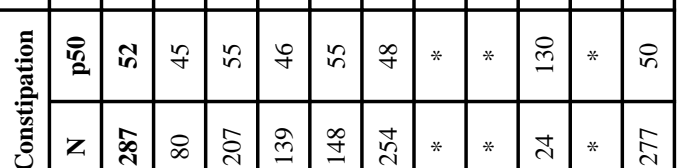

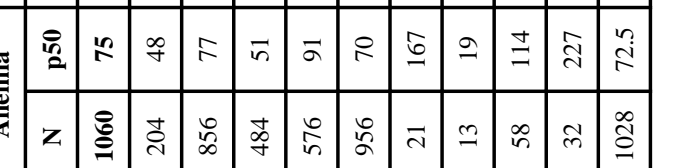

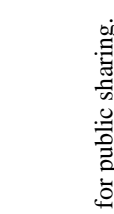

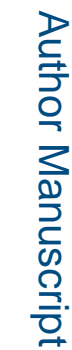
密 $\frac{\sqrt{\frac{2}{0}}}{\frac{2}{2}}$ 氧

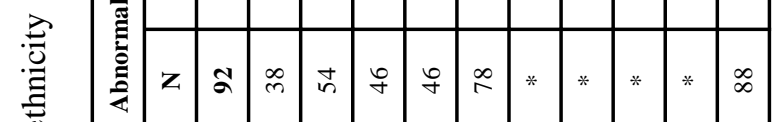
要 
Table 4.\#

Multivariate logistic regression of characteristics associated with endoscopy completion after a billed CRCassociated symptom

\begin{tabular}{|c|c|c|c|}
\hline & $\mathrm{aOR}^{*}$ & $95 \% \mathrm{CI}$ & p-value \\
\hline \multicolumn{4}{|l|}{ Gender } \\
\hline Male & Ref. & -- & -- \\
\hline Female & 1.08 & $0.89-1.30$ & 0.44 \\
\hline \multicolumn{4}{|l|}{ Race } \\
\hline White & Ref. & -- & -- \\
\hline AA & 1.57 & $0.77-3.22$ & 0.79 \\
\hline $\mathrm{AI} / \mathrm{AN}$ & 1.23 & $0.47-3.22$ & 0.70 \\
\hline Asian/PI & 1.63 & $1.0-2.64$ & 0.63 \\
\hline \multicolumn{4}{|l|}{ Ethnicity } \\
\hline Non-Hispanic & Ref. & -- & -- \\
\hline Hispanic & 1.40 & $0.76-2.60$ & 0.28 \\
\hline \multicolumn{4}{|l|}{ RUCA } \\
\hline Metropolitan Area & Ref. & -- & -- \\
\hline Metro w/ commute & 1.10 & $0.82-1.49$ & 0.97 \\
\hline Micropolitan Area & 0.79 & $0.58-1.07$ & 0.98 \\
\hline Small town/Rural & 0.87 & $0.60-1.25$ & 0.97 \\
\hline \multicolumn{4}{|l|}{ Living Arrangement } \\
\hline Living w/o partner & Ref. & -- & -- \\
\hline Living w/ partner & 1.52 & $1.23-1.86$ & 0.01 \\
\hline Unknown & 1.33 & $0.98-1.80$ & 0.60 \\
\hline \multicolumn{4}{|l|}{ Income Quartile } \\
\hline Q1 & Ref. & -- & -- \\
\hline Q2 & 1.07 & $0.82-1.40$ & 0.97 \\
\hline Q3 & 0.94 & $0.71-1.23$ & 0.97 \\
\hline Q4 & 0.82 & $0.62-1.08$ & 0.97 \\
\hline \multicolumn{4}{|l|}{ Insurance Type } \\
\hline Commercial & Ref. & -- & -- \\
\hline Medicaid & 0.77 & $0.44-1.34$ & 0.78 \\
\hline Medicare & 0.63 & $0.48-0.82$ & 0.01 \\
\hline \multicolumn{4}{|l|}{ Charlson Score } \\
\hline 0 & Ref. & -- & -- \\
\hline 1 & 0.95 & $0.74-1.23$ & 0.29 \\
\hline $2+$ & 0.71 & $0.57-0.88$ & $<0.01$ \\
\hline
\end{tabular}

AA- African American; AI/AN- American Indian/Alaska Native; Asian/PI- Asian/Pacific Islander; RUCA - Rural-Urban Commuting Area Codes *

Adjusted for gender, race, ethnicity, insurance, living arrangement, Charlson Comorbidity, RUCA, and socioeconomic status 
\# Per CMS rules, cells with $\mathrm{N}<11$ must be censored/collapsed into another category or coarsened appropriately for public sharing. Some groups categories as N/A, unknown or other are not shown in this table due to low patient counts. 